\title{
Low-Cost Eye Phantom for Stereophotogrammetry- Based Optic Nerve Head Topographical 3D Imaging
}

Ian Coghill

Richard A Black

lain A T Livingstone

Mario E Giardini

I. Coghill, R. A. Black, I. A. T. Livingstone and M. E. Giardini, "Low-Cost Eye Phantom for Stereophotogrammetry-Based Optic Nerve Head Topographical 3D Imaging," 2020 42nd Annual International Conference of the IEEE Engineering in Medicine \& Biology Society (EMBC), Montreal, QC, Canada, 2020, pp. 1604-1607, doi: 10.1109/EMBC44109.2020.9175326.

This is an author-generated copy of the accepted version.

(C) 2020 IEEE. Personal use of this material is permitted. Permission from IEEE must be obtained for all other uses, in any current or future media, including reprinting/republishing this material for advertising or promotional purposes, creating new collective works, for resale or redistribution to servers or lists, or reuse of any copyrighted component of this work in other works. 


\title{
Low-Cost Eye Phantom for Stereophotogrammetry-Based Optic Nerve Head Topographical 3D Imaging
}

\author{
Ian Coghill, Student Member, IEEE-EMBS, Richard A. Black, Iain A.T. Livingstone and Mario E. \\ Giardini, Member, IEEE-EMBS
}

\begin{abstract}
Glaucoma is the second leading cause of blindness globally. Stereophotogrammetry-based optic nerve head topographical imaging systems could potentially allow for objective glaucoma assessment in settings where technologies such as optical coherence tomography and the Heidelberg Retinal Tomograph are prohibitively expensive. In the development of such systems, eye phantoms are invaluable tools for both system calibration and performance evaluation. Eye phantoms developed for this purpose need to replicate the optical configuration of the eye, the related causes of measurement artefacts, and give the possibility to present to the imaging system the targets required for system calibration. The phantoms in the literature that show promise of meeting these requirements rely on custom lenses to be fabricated, making them very costly. Here, we propose a low-cost eye phantom comprising a vacuum formed cornea and commercially available stock bi-convex lens, that is optically similar to a gold-standard reference wide-angle schematic eye model and meets all the compliance and configurability requirements for use with stereo-photogrammetry-based $\mathrm{ONH}$ topographical imaging systems. Moreover, its modular design, being fabricated largely from 3D-printed components, lends itself to modification for other applications. The use of the phantom is successfully demonstrated in an ONH imager.
\end{abstract}

\section{INTRODUCTION}

The second leading cause of blindness globally is glaucoma [1], an optic neuropathy that causes gradual and irreversible vision loss, typically asymptomatic until advanced. Vision loss can be prevented if detected and treated early [2]. Enlargement of the cup of the optic nerve head $(\mathrm{ONH})$, due to loss of neural tissue, can precede detectable vision loss and as such is a useful indicator for early detection of the condition [3]. Assessment of the $\mathrm{ONH}$, ordinarily by subjective examination of stereoscopic $\mathrm{ONH}$ images by a specialist [4], is not always possible, especially in low-income countries [5]. Where specialists cannot be present, quantitative imaging technologies that can provide an objective assessment of the ONH, such as optical coherence tomography (OCT) and the Heidelberg Retinal Tomograph (HRT), could be advantageous [6] but their high cost is prohibitive in most middle- and low-income settings and, even in high-income settings (such as community based optometry), only marginally suitable for mass population screening. Quantitative $\mathrm{ONH}$ imaging systems based on stereophotogrammetry, such as we described in previous work [7], could potentially be a cost-effective alternative.

*Research supported by the UK EPSRC (Grant Ref. EP/L015595/1).

I. Coghill, R. A. Black and M.E. Giardini are with the Department of Biomedical Engineering, University of Strathclyde, Glasgow G1 1XQ, UK (e-mail: ian.coghill@strath.ac.uk).
In the development of such systems, eye phantoms play a crucial role in calibration and performance evaluation. Eye phantoms have previously been reported by researchers working in this area [7], [8]. In both cases, the eye phantoms were very simplistic: they contained a single lens for refractive power, were air-filled and were not true to scale, thus incorrectly mimicking artefacts and aberration, hence of dubious validity for testing imaging devices designed for human eyes. More optically correct eye phantoms, fluid-filled, life-sized and containing two lenses to emulate the cornea and crystalline lens [9]-[11] have been developed for other purposes. These eye phantoms contained custom lenses, at very high cost and complexity.

Considering this, the current work proposes a low-cost and mostly 3D-printable eye phantom containing a cornea that, while tightly complying with the reference model eye by Navarro [12] can be created using a vacuum forming machine and, to emulate the crystalline lens, a commercially available stock bi-convex lens. It was designed specifically for use with stereophotogrammetry-based $\mathrm{ONH}$ topographical imaging systems, however it is possible to modify the design for use in other areas. We demonstrate also the imaging of the phantom with an upgraded version of the stereophotogrammetry-based ONH topographical imaging system described in our previous work [7].

\section{METHOD}

The optical design (described in Section A, below) of the eye phantom was carried out first, followed by the mechanical design and fabrication (Section B). Following fabrication, its use in calibration and evaluation was demonstrated (Section C).

\section{A. Optical Design}

The optical design of the eye phantom was based on a schematic eye model. A number of different schematic eye models exist, with complexity ranging from those with a single refracting surface to those with two refracting surfaces for the cornea and a gradient-index crystalline lens [13]. Navarro's wide-angle schematic eye model [12], which well balances optical performance and complexity, was selected as the model to base the optical design on, as was done by other researchers in the design of their eye phantoms [9]-[11]. Importantly, this model accurately accounts for the optical performance of the average adult human eye off-axis, which is appropriate for $\mathrm{ONH}$ related work as the $\mathrm{ONH}$ is located at

I. A.T. Livingstone is with NHS Forth Valley, Falkirk Community Hospital, Falkirk FK1 5QE, UK (email: iain.livingstone@nhs.net). 
around $10^{\circ}$ off the axis of the lens [14]. The optical data for Navarro's eye model are shown in the first four columns of Table 1.

Implementing Navarro's eye model exactly in an eye phantom is challenging. Bakaraju et al. [10] were able to create an eye phantom that contained a custom lathed fluoro-polymer cornea and diamond-turned Boston RGP polymer lens. Corcoran et al. [9] were likewise able to create an accurate eye phantom by using a custom fused silica cornea and $\mathrm{CaF}_{2}$ lens. The phantom by Xie and colleagues [11] contained a custom polymethyl methacrylate (PMMA) cornea and an intraocular lens (IOL). In our case, given the high cost of custom lens manufacture, the undesirable limitations to the pupil size imposed by IOLs (typically $6 \mathrm{~mm}$ ), and the scarce availability of IOL optical simulation data, it was decided to fabricate the cornea by vacuum forming a clear plastic sheet over a ball bearing, and to use a stock bi-convex lens to imitate the crystalline lens.

The cornea of the eye phantom was designed to match, as closely as possible, the refractive power of the cornea of Navarro's eye model. It was decided to use High Impact Polystyrene (HIPS, $\mathrm{n}=1.5915$ at $589.3 \mathrm{~nm}$ [15]), as the cornea needs to operate while immersed in a liquid. HIPS is nonhygroscopic, and the alteration of optical and mechanical properties by the fluid interior of the eye phantom is low compared to most transparent plastics. Furthermore, HIPS has excellent optical quality and is readily available in a thickness of $0.5 \mathrm{~mm}$, close to the $0.55 \mathrm{~mm}$ central thickness of Navarro's eye model. The use of vacuum forming to fabricate the cornea imposed some constraints on its optical design. The cornea had to be of uniform thickness, however, as the thickness of the cornea of Navarro's eye model does not deviate much from uniformity, this was not considered to represent a major limitation. The radius of the posterior surface had to match that of a widely available forming mold, in our case effectively a ball bearing. The power of the cornea in Navarro's eye model was determined to be +42.88 diopters, using the equations described by Xie and co-workers [11]. It was determined that a uniform thickness HIPS cornea with anterior and posterior radii of $7.5 \mathrm{~mm}$ and $7 \mathrm{~mm}$, respectively, achievable by vacuum forming a $0.5 \mathrm{~mm}$ thick HIPS sheet over a $14 \mathrm{~mm}$ diameter ball bearing, would have a power of +42.77 diopters, hence this radius was chosen for the design.

The specific lens was chosen by modelling and simulating (at a single $589.3 \mathrm{~nm}$ wavelength) the performance of eye phantoms containing the HIPS cornea and various commercially available stock bi-convex lenses. All simulations were performed with the optical design software
OpticStudio 14.2 (Zemax Europe Ltd, Bedford, UK) and the results were compared with those obtained with Navarro's eye model to determine which stock lens resulted in the closest match in terms of resolution, axial length and distortion. Resolution and distortion were recorded between field angles of $0^{\circ}$ and $25^{\circ}$. In each case the anterior chamber depth and radius of the retina was set to be the same as Navarro's eye model. The iris diameter in each case was set to $8 \mathrm{~mm}$, as was the iris in the implementation of Navarro's eye model. Water was to be used to fill the interior of the eye phantom and as such the refractive index of the aqueous and vitreous humor was set to 1.3324 , which corresponds to that of water at 589.3 $\mathrm{nm}$ and $25^{\circ} \mathrm{C}$ [16]. The distance between the posterior surface of the lens and the retina was adjusted to minimize the RMS spot size. The simulations determined that the eye phantom best matching Navarro's eye model contained a lens which had an effective focal length of $18 \mathrm{~mm}$ and a diameter of $9 \mathrm{~mm}$ (32968, Edmund Optics Ltd, York, UK). The final optical design of the complete eye phantom is described by the optical data provided in the rightmost four columns of Table 1. (Note that the surfaces in the eye phantom are spherical only, which is why a value of zero has been set for the $Q$ value of each of the surfaces.)

\section{B. Mechanical Design and Fabrication}

The eye phantom has two configurations: an imaging configuration and a calibration configuration. Both configurations use the same anterior section but different interchangeable posterior sections. The main structure of these sections of the eye phantom were printed in EnvisionTEC ABS Tough on a Perfactory Aureus 3D printer (EnvisionTEC, Stoke-on-Trent, UK). The 3D models of all 3D printed parts were generated using the CAD software Rhinoceros 6 (Robert McNeel \& Associates, USA). In both configurations the eye phantom was filled with water. 3D models (sectional) and images of the eye phantom in its two configurations are provided in Fig. 1.

The anterior section of the eye phantom featured the cornea, iris and lens. As per the previous section, the cornea was fabricated by vacuum forming a clear $0.5 \mathrm{~mm}$ HIPS sheet (4D Modelshop Ltd, London, UK) over a $14 \mathrm{~mm}$ diameter grade 100 hardened 52100 chrome steel ball bearing (Simply Bearings Ltd, Leigh, UK) using a dental vacuum forming machine (Jintai, Zhejiang, China). The cornea was cut down to a diameter of $\sim 13.5 \mathrm{~mm}$ before being secured to the anterior section with LOCTITE® 4305 UV curable glue (Loctite, Dusseldorf, Germany). The bi-convex lens was placed in the anterior chamber, up against the $0.35 \mathrm{~mm}$ thick $8 \mathrm{~mm}$ iris, and

TABLE I. OPTICAL DATA FOR NAVARRO’S WIDE-ANGLE SCHEMATIC EYE MODEL AND THE PROPOSED EYE PHANTOM

\begin{tabular}{|c|c|c|c|c|c|c|c|c|}
\hline \multirow[b]{2}{*}{ Surface } & \multicolumn{4}{|c|}{ Navarro's Wide-Angle Schematic Eye Model } & \multicolumn{4}{|c|}{ Eye Phantom } \\
\hline & Radius (mm) & $\begin{array}{c}\text { Thickness } \\
(\mathrm{mm})\end{array}$ & $Q$ & $\begin{array}{l}\text { Refractive Index } \\
\text { (@ } 589.3 \text { nm) }\end{array}$ & Radius (mm) & $\begin{array}{c}\text { Thickness } \\
(\mathrm{mm})\end{array}$ & $Q$ & $\begin{array}{c}\text { Refractive Index } \\
\text { (@ } 589.3 \text { nm) }\end{array}$ \\
\hline 1 & 7.72 & 0.55 & -0.26 & 1.376 & 7.5 & 0.5 & 0 & 1.5915 \\
\hline 2 & 6.5 & 3.05 & 0 & 1.3374 & 7 & 3.05 & 0 & 1.3324 \\
\hline Iris & $\infty$ & 0 & - & 1.3374 & $\infty$ & 0 & - & 1.3324 \\
\hline 3 & 10.2 & 4 & -3.1316 & 1.42 & 18.15 & 2.6 & 0 & 1.5167 \\
\hline 4 & -6 & 16.3203 & -1 & 1.336 & -18.15 & 17.465 & 0 & 1.3324 \\
\hline 5 & -12 & - & 0 & - & -12 & - & 0 & - \\
\hline
\end{tabular}




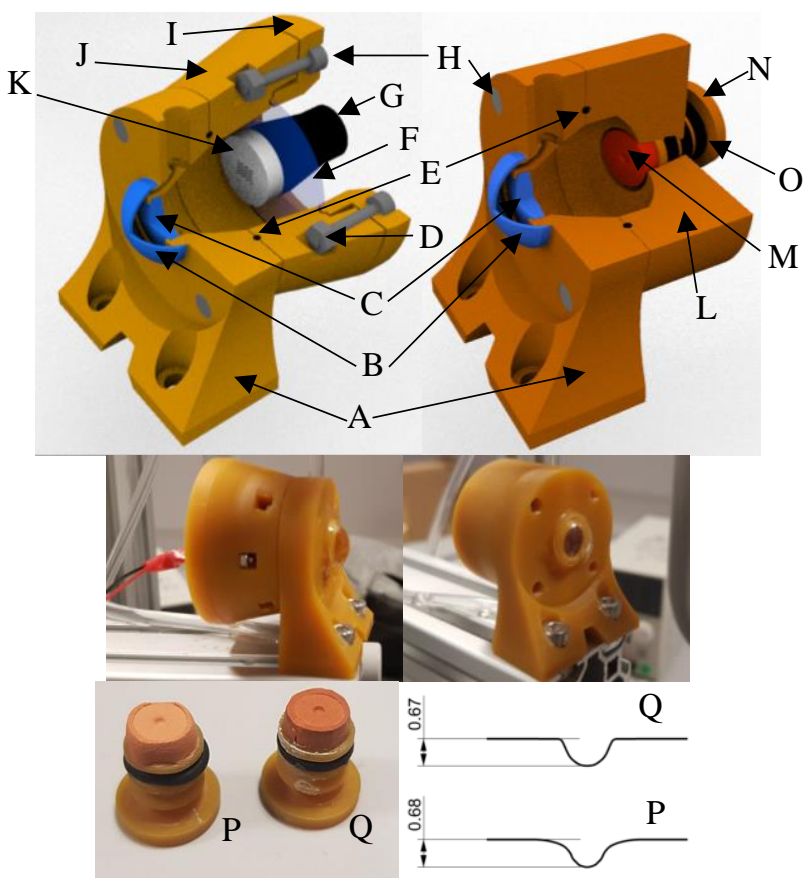

Figure 1. Sectional 3D model of the eye phantom in its calibration (top left) and imaging (top right) configurations; Images of the eye phantom in its calibration (middle left) and imaging (middle right) configurations; Optic nerve head models (bottom left) and their corresponding half crosssectional profiles (bottom right). A - Anterior Section, B - HIPS Cornea, C - Bi-Convex Lens, D - M2 Nut, E - Nitrile Rubber O-Ring, F - Nitrile Rubber Sheet, G - LED Mount, H - M2 Bolt, I - Posterior Section for Calibration Part 1, J - Posterior Section for Calibration Part 2, K -

Calibration Pattern, L - Posterior Section for Imaging, M - Terracotta ONH Model, N - ONH Model Mount, O - Nitrile Rubber O-Ring, P Normal ONH model, Q - Glaucomatous ONH Model.

secured with a small amount of the same glue. The anterior section has holes at its top and bottom which lead to channels to enable water to flow into the eye phantom's anterior chamber and posterior cavity. Two $50 \mathrm{ml}$ disposable syringes (Dieckhoff \& Ratschow Praxisdienst GmbH \& Co.KG, Longuich Germany) were connected to these holes via IV extension tubes (Dieckhoff \& Ratschow Praxisdienst GmbH \& Co.KG, Longuich Germany) to enable water injection into the eye phantom. The posterior sections for the two configurations of the eye phantom is secured onto the anterior section using M2 bolts and M2 nuts (VIGRUE, Guangdong, China) and sealed using a nitrile rubber O-ring (SourcingMap, Hong Kong, China).

Two ONH models were created - a glaucomatous and a normal ONH model. These were machined from Terracotta using a CNC engraving machine (Shenzhen Ni Xing Ni Shang Electronic Commerce Co Ltd, Shenzhen, China) with a $10^{\circ}$, $0.1 \mathrm{~mm}$ diameter engraving bit (Man Hing Steel Iron Engineering Company, Hong Kong, China). The shape of the ONH models was generated in the same manner as in our previous work [7] but had a different scale, as appropriate for this life-sized eye phantom. The ONH models, on the mounts, are depicted in Fig. 1 together with the half cross-sectional profiles of their top surfaces. The posterior section of the eye phantom in its imaging configuration holds the ONH models in the appropriate location and allows for adjustment of the distance between the $\mathrm{ONH}$ models and the bi-convex lens. The target location for the center of the $\mathrm{ONH}$ models is at $10^{\circ} \mathrm{left}$ off-axis horizontally and $1.5^{\circ}$ upwards to match $\mathrm{ONH}$ position in the human left eye [14]. It is possible, by mirroring the design of this section to switch the $\mathrm{ONH}$ position to that which would be seen for the right eye. The ONH models were glued onto ONH model mounts using UV curable glue. These mounts were press fit into the main part of this section and the seal was made using nitrile rubber O-rings (Zhongbo Business Limited, Hong Kong, China).

In its calibration configuration, the posterior section of the eye phantom allows for a calibration pattern, in our case a flat checkerboard, to be held in different positions and orientations inside the eye phantom at the location of the retina. This section is backed by a thin nitrile rubber sheet, cut from nitrile gloves (Supermax Healthcare Ltd, Peterborough, UK), clamped between the phantom body and a retaining ring. The checkerboard calibration pattern was created photographically on Rollei RPX black and white film (Rollei, Hamburg, Germany), comprising $10 \times 70.392$ mm black and white squares. The film was glued flat on to a cast white acrylic (Simply Plastics Ltd, Essex, UK) disc, $12 \mathrm{~mm}$ in diameter and $3 \mathrm{~mm}$ thick, using the UV curable glue. 4 neodymium magnets, $2 \mathrm{~mm}$ in diameter and $1 \mathrm{~mm}$ thick, were glued to the opposite face of the acrylic disc using the same glue. On the other side of the nitrile sheet was the illumination section, magnetically attached to the calibration target using 4 of the same magnets. The illumination section featured a mount, 3D printed in black PLA (colorFabb B.V., Belfeld, Netherlands) on the fused deposition modelling printer Ultimaker 2+ (Ultimaker B.V., Geldermalsen, Holland), for a $5 \mathrm{~mm}$ white LED (RS Components Ltd, Corby, UK). Electrical wires were soldered on to the LED to allow a power source to be connected to it. It must be noted that, during calibration of the imaging system, anatomical compliance is not relevant, and therefore target retroillumination was adopted to avoid any difficulties with reflections from the shiny surface of the film.

\section{Eye Phantom Use Demonstration}

The stereophotogrammetry-based topographical optic nerve head $(\mathrm{ONH})$ imaging system from our previous work [7], consisting of a slit lamp modified to deliver binocular images to an appropriate processing algorithm, was used to capture stereo image pairs of the normal and glaucomatous $\mathrm{ONH}$ models of the fabricated eye phantom. Here, we marginally updated the processing algorithm to divide the region of interest into $20 \times 20$ blocks instead of $15 \times 15$ which we estimate are necessary in order to cover the optic nerve head area with a number of points more appropriate for future clinical work. Twenty-two calibration image pairs were used. Thereafter, the ONH models were scanned by a 3D optical surface measurement system (Alicona Infinite Focus IFM G4, Alicona Imaging $\mathrm{GmbH}$, Graz, Austria), providing the accurate ground truth scans against which the 3D reconstructions were validated.

\section{RESUlTS AND DISCUSSION}

The optical simulations established that, for successful imaging using our cornea and lens, the design axial length of the eye phantom was $23.62 \mathrm{~mm}$, very close to that of Navarro's eye model (23.92 mm). In terms of distortion, it can be seen in Fig. 2 that the percentage of distortion is very similar between the eye phantom and Navarro's eye model at all tested field angles. Furthermore, in terms of resolution, again as per Fig. 2, the RMS spot size at all field angles up to 
$20^{\circ}$ is similar between the two, however drastically increasing at $25^{\circ}$. While surfaces 3 and 4 deviate significantly from Navarro's model, this is due to the use of stock lenses rather than custom optics and, indeed, this may be the cause of the drastic degradation of performance at $25^{\circ}$. Future optimization will necessarily need to take this into account, possibly again using stock lenses to contain costs. Nevertheless, the matching of the imaging properties of the eye phantom with Navarro's eye model is deemed sufficient for studies involving the $\mathrm{ONH}$ which is small (around $5^{\circ}$ wide) and centered at $\sim 10^{\circ}$ off-axis [14]. Also shown in Fig. 2 are the cross-sectional profiles of co-aligned 3D reconstructions and their corresponding ground truths, which show excellent matching. Statistical quantification of the deviation is underway.

As the cost of the complex eye phantoms developed by others [9]-[11] is not reported, it was not possible to do a comparison of cost. However, to our knowledge, the custom optics required by the literature phantoms amount to several thousands of US dollars. In this sense, we estimate that the cost of the eye phantom created in this work is likely to be an order of magnitude lower. Moreover, it is relatively straightforward to fabricate, and it can be easily adapted for use in other ophthalmic imaging studies. To facilitate this, the design files have been made publicly available at https://github.com/biomeddev/simple-eye-phantom under a Creative Commons Attribution 4.0 International Public License ("CC BY 4.0") [17] and will be continuously updated. A snapshot of the repository at the time of publication is also available on the University of Strathclyde repository for longterm availability, locatable through the following DOI: 10.15129/89e9032d-18c8-44ff-a28e-e964e5595fbc.
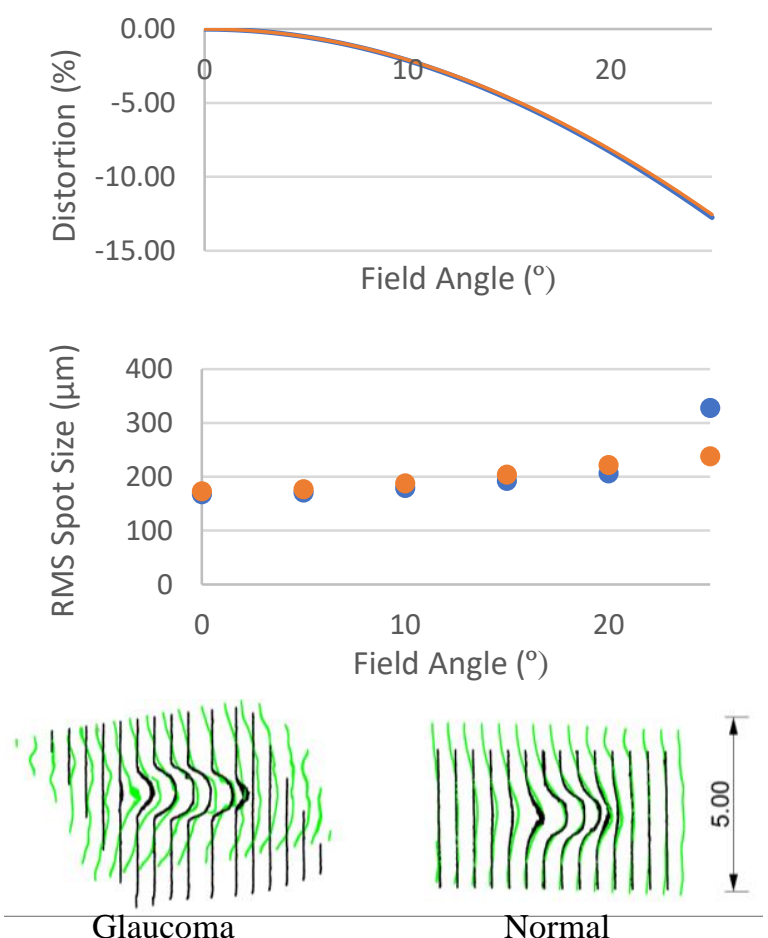

Figure 2. Top: distortion vs field angle for Navarro's eye model (orange) and the eye phantom (blue); Middle: RMS Spot Size at various field angles for Navarro's eye model (orange) and the eye phantom (blue); Bottom: Cross-sectional profiles from the aligned 3D reconstructions and their corresponding ground truth (green: 3D reconstruction, black: ground truth).

\section{CONCLUSION}

This work presents an optically-accurate eye phantom that can be fabricated at low cost. It was shown to behave similar to Navarro's eye model in terms of optical performance at least until a field angle of $20^{\circ}$. Its use was successfully demonstrated on a stereophotogrammetry-based $\mathrm{ONH}$ topographical imaging system.

\section{REFERENCES}

[1] S. Kingman, 'Glaucoma is second leading cause of blindness globally.', Bull. World Health Organ., vol. 82, no. 11, p. 887, Nov. 2004.

[2] J. B. Jonas, T. Aung, R. R. Bourne, A. M. Bron, R. Ritch, and S. Panda-Jonas, 'Glaucoma', The Lancet, vol. 390, no. 10108, pp. 2183-2193, Nov. 2017, doi: 10.1016/S0140-6736(17)31469-1.

[3] S. J. Mckinnon, 'The Value of Stereoscopic Optic Disc Photography', p. 3, 2005.

[4] J. A. Giaconi, S. K. Law, K. Nouri-Mahdavi, A. L. Coleman, and J. Caprioli, Eds., Pearls of Glaucoma Management, 2nd ed. Berlin Heidelberg: Springer-Verlag, 2016.

[5] R. Thomas, 'Glaucoma in developing countries', Indian J. Ophthalmol., vol. 60, no. 5, pp. 446-450, 2012, doi: 10.4103/03014738.100546.

[6] R. M. Vessani, R. Moritz, L. Batis, R. B. Zagui, S. Bernardoni, and R. Susanna, 'Comparison of quantitative imaging devices and subjective optic nerve head assessment by general ophthalmologists to differentiate normal from glaucomatous eyes', J. Glaucoma, vol. 18, no. 3, pp. 253-261, Mar. 2009, doi: 10.1097/IJG.0b013e31818153da.

[7] I. Coghill, K. C. Jordan, R. A. Black, I. A. T. Livingstone, and M. E. Giardini, '3D reconstruction of the optic nerve head of a phantom eye from images obtained using a slit lamp fitted with low cost add-ons', presented at the 41st Annual International Conference of the IEEE Engineering in Medicine and Biology Society (EMBC), Jul. 2019, Accessed: Sep. 18, 2019. [Online]. Available: https://pureportal.strath.ac.uk/en/publications/3d-reconstruction-ofthe-optic-nerve-head-of-a-phantom-eye-from-i.

[8] T. Nakagawa et al., 'Quantitative depth analysis of optic nerve head using stereo retinal fundus image pair', J. Biomed. Opt., vol. 13, no. 6, p. 064026, Dec. 2008, doi: 10.1117/1.3041711.

[9] A. Corcoran, G. Muyo, J. van Hemert, A. Gorman, and A. R. Harvey, 'Application of a wide-field phantom eye for optical coherence tomography and reflectance imaging', J. Mod. Opt., vol. 62, no. 21, pp. 1828-1838, Dec. 2015, doi: 10.1080/09500340.2015.1045309.

[10] R. C. Bakaraju, K. Ehrmann, D. Falk, A. Ho, and E. Papas, 'Physical human model eye and methods of its use to analyse optical performance of soft contact lenses', Opt. Express, vol. 18, no. 16, pp. 16868-16882, Aug. 2010, doi: 10.1364/OE.18.016868.

[11] P. Xie et al., 'Application of 3-Dimensional Printing Technology to Construct an Eye Model for Fundus Viewing Study', PLOS ONE, vol. 9, no. 11, p. e109373, Nov. 2014, doi: 10.1371/journal.pone.0109373.

[12] I. Escudero-Sanz and R. Navarro, 'Off-axis aberrations of a wideangle schematic eye model', J. Opt. Soc. Am. A Opt. Image Sci. Vis., vol. 16, no. 8, pp. 1881-1891, Aug. 1999, doi: 10.1364/josaa.16.001881.

[13] D. A. Atchison and L. N. Thibos, 'Optical models of the human eye', Clin. Exp. Optom., vol. 99, no. 2, pp. 99-106, Mar. 2016, doi: 10.1111/cxo.12352.

[14] D. A. Atchison and G. Smith, Optics of the Human Eye. Elsevier, 2000.

[15] N. Sultanova, S. N. Kasarova, and I. Nikolov, 'Dispersion Properties of Optical Polymers', 2009, doi: 10.12693/aphyspola.116.585.

[16] G. M. Hale and M. R. Querry, 'Optical Constants of Water in the 200nm to 200- $\mu \mathrm{m}$ Wavelength Region', Appl. Opt., vol. 12, no. 3, pp. 555-563, Mar. 1973, doi: 10.1364/AO.12.000555.

[17] 'Creative Commons - Attribution 4.0 International - CC BY 4.0'. https://creativecommons.org/licenses/by/4.0/ (accessed Jan. 20, 2020). 